\title{
An Innovative Genetic Algorithms-Based Inexact Non-Linear Programming Problem Solving Method
}

\author{
Weihua Jin, Zhiying Hu, Christine Chan \\ Energy Informatics Laboratory, Faculty of Engineering \& Applied Science. University of Regina, Regina, SK, Canada \\ Email: Zhiying.hu@uregina.ca
}

How to cite this paper: Jin, W.H., Hu, Z.Y. and Chan, C. (2017) An Innovative Genetic Algorithms-Based Inexact Non-Linear Programming Problem Solving Method. Journal of Environmental Protection, 8, 231-249.

https://doi.org/10.4236/jep.2017.83018

Received: October 13, 2016

Accepted: March 11, 2017

Published: March 14, 2017

Copyright ( 92017 by authors and Scientific Research Publishing Inc. This work is licensed under the Creative Commons Attribution International License (CC BY 4.0). http://creativecommons.org/licenses/by/4.0/

\begin{abstract}
In this paper, an innovative Genetic Algorithms (GA)-based inexact non-linear programming (GAINLP) problem solving approach has been proposed for solving non-linear programming optimization problems with inexact information (inexact non-linear operation programming). GAINLP was developed based on a GA-based inexact quadratic solving method. The Genetic Algorithm Solver of the Global Optimization Toolbox (GASGOT) developed by MATLAB $^{\mathrm{TM}}$ was adopted as the implementation environment of this study. GAINLP was applied to a municipality solid waste management case. The results from different scenarios indicated that the proposed GA-based heuristic optimization approach was able to generate a solution for a complicated nonlinear problem, which also involved uncertainty.
\end{abstract}

\section{Keywords}

Genetic Algorithms, Inexact Non-Linear Programming (INLP), Economy of Scale, Numeric Optimization, Solid Waste Management

\section{Introduction}

Municipal solid waste management involves activities such as waste collection, transportation, treatment, reutilization, and disposal. Economic optimization in the operation programming of solid waste management was first proposed in the 1960s [1]. Different models of waste planning have been researched and applied in various engineering fields in the following decades. The primary considerations involved are cost control, environmental sustainability and waste reutilization. The techniques employed include linear programming [2] [3] [4] [5], mixed integer linear programming [6], multi-objective programming [7] [8] [9], nonlinear programming [10] [11], as well as their hybrids, which involve probability, fuzzy set 
and inexact analysis [12] [13] [14] [15] [16]. Due to complexity of the problem, research reports on nonlinear programming problems for solid waste management are scarce; some exceptions are [17] [18]. In some of the works such as [10] [11], the nonlinear objective functions are converted into linear functions or simplified into quadratic functions under some adopted conditions and assumptions.

The approach of operational programming with inexact analysis often treats the uncertain parameters as intervals with known lower and upper bounds and unclear distributions. A major advantage of inexact programming is that the variation in system performance and decision variables can be investigated by solving relatively simple sub-models. In real-life problems, while the available information is often inadequate and the distribution functions are often unknown, it is generally possible to represent the obtained data with inexact numbers that can be readily used in the inexact programming models. For decision makers, it is usually more feasible to represent uncertain information as inexact data than to specify distributions of fuzzy sets or probability functions. Hence, various kinds of inexact programmings such as inexact linear programming (ILP), inexact quadratic programming (IQP), inexact integer programming (IIP), inexact dynamic programming (IDP) and inexact multi-objective programming (IMOP) have been developed and are well discussed [10] [11] [19] [20] [21] [22]. It can be observed from these studies that applications of inexact models to practical solid waste planning systems are effective.

In [23], the approach of GA for ILP and IQP is discussed; the comparisons of traditional binary analysis solving methods [8] [21] [22] [24] with GA-based methods indicate that for ILP and IQP, GA can generate better results with less computational complexity.

In the literature, much work on traditional binary analysis for IQP has been done, for example, see [10] [11] [21] [22]. However, traditional binary analysis methods for ILP and IQP involve unavoidable simplifications and assumptions, which often increased the chance for error in the problem solving process and adversely affected the quality of the results. Moreover, a more complex model often increases error in the solution. However, it has been observed that more complex models often produce less optimal results, and studies that focus on inexact nonlinear programming problems are scarce. For example, in [19], the methodology is mainly focused on combining endpoint values of the inexact parameters to form a set of deterministic problems, which will only work for particular monotone functions within a small scale model. Therefore, a more flexible problem solving method for the general INLP is desired.

In this paper, we propose to use GA as the optimization technique for solving complex and non-linear problems in operations research, industrial engineering and management science. The GA method is a suitable optimization approach especially for solving problems that involve non-smooth and multi-modal search spaces. The innovative GA-based inexact non-linear programming (GAINLP) solving approach is useful for solving non-linear programming problems with inexact information, or inexact non-linear operation programming problems. 
This paper is organized as follows. Section 2 presents the background of the research, which includes an introduction to the problem of Solid Waste Management (SWM), the concept of economies of scale in SWM, and the concept of GA, and the Genetic Algorithm Non-Linear Solver Engine (GANLP) that is used for implementing the proposed method. Section 3 discusses the methodology of the proposed GA-based methods for solving inexact non-liner problems. Section 4 presents a case study in which the solutions for different scenarios of the INLP problem of solid waste disposal planning are generated.

\section{Background}

\subsection{Solid Waste Management and the Concept of Economy of Scale in Solid Waste Management Planning}

Solid waste management is the process of removing waste materials from the surrounding environment, which involves the collection, separation, storage, processing, treatment, transport, recovery and disposal of solid waste. Landfill and incineration are two of the most commonly used solid waste disposal methods. The objective of a solid waste management process is to dispose of discarded materials in a timely manner so as to prevent the spread of disease, minimize the likelihood of contamination, and reduce their effects on human health and the environment.

The economy of scale (ES) is a microeconomics term, and it refers to the advantages that enterprises obtain due to their size or scale of operation, with the cost per unit of output generally decreasing as the scale increases and fixed costs are distributed over more units of output. In a solid waste management system, ES exists within the transportation process [25] and it can be expressed as a sizing model with a power law [11].

$$
C_{t}=C_{r e}\left(X_{t} / X_{r e}\right)^{1+m}
$$

where $X_{t}(\mathrm{t} / \mathrm{d})$ is a waste flow decision variable; $X_{r e}(\mathrm{t} / \mathrm{d})$ is a reference waste flow; $C_{t}(\$ / \mathrm{t})$ is the transportation unit cost due to the ES of waste flow $X_{t}(\mathrm{t} / \mathrm{d}) ; C_{r e}(\$ / \mathrm{t})$ is a coefficient reflecting the significance of the economy of scale to the unit cost of waste transportation for reference waste flow $X_{r e}(\mathrm{t} / \mathrm{d})$, $C_{r e}<0$; and $m$ is an economy of scale exponent which reflects the unit cost decline with respect to the waste flow, $-1<m<0$.

Thus the cost function for waste transportation can be expressed as,

$$
C=X_{t}\left(A+C_{t}\right)
$$

where $C(\$)$ is the transportation cost due to the variable $X_{t}, A(\$ / t)$ is a fixed unit charge, and $A+C_{t}$ is the unit transportation cost. The unit transportation cost affected by ES is shown in Figure 1.

By introducing ES into a large-scale solid waste management model, the transportation procedure can be simulated more accurately. At the same time, the incorporation of ES adds a nonlinear factor into the objective function, and hence, more computational effort for problem solving will be required. Within this context, we propose GA as a good problem solving method for this type of problems. 


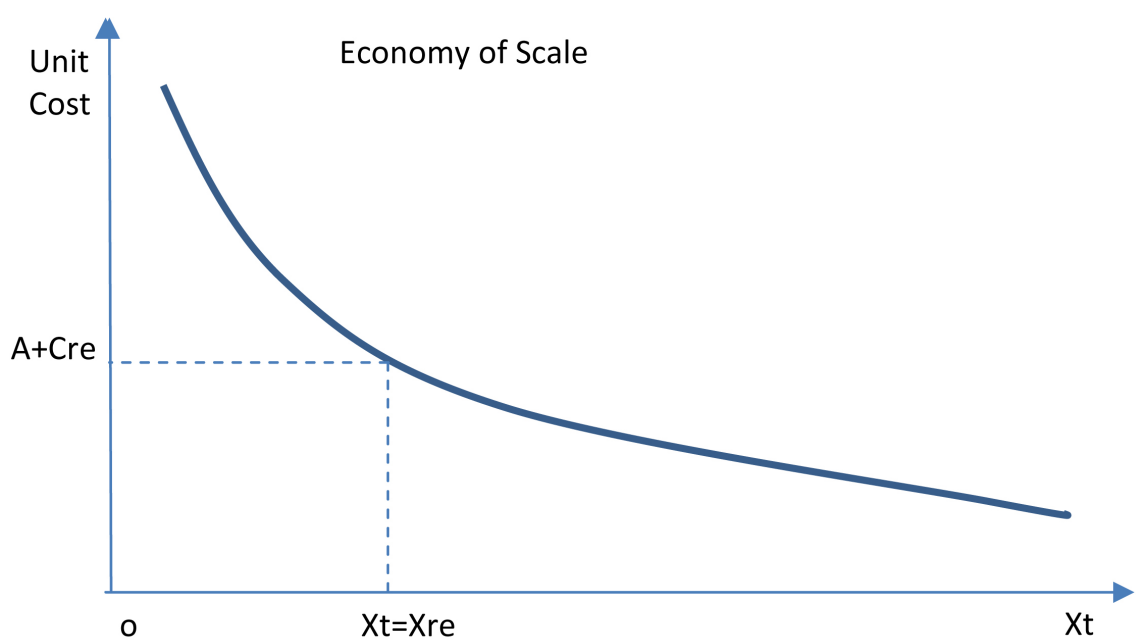

Figure 1. Unit transportation cost affected by ES.

In the next section, the concept of GA is introduced.

\subsection{GA for Solving Non-Linear Problems (GANLP)}

\subsubsection{GA for Deterministic Optimization Problems}

A generic procedure of GA can be summarized as follows:

1) Initialization: The initialization step involves establishing the mapping mode between the genotype and phenotype; this can be done by determining the coding and decoding functions, creating the fitness function according to the objective of the problem domain, and generating the initial population $\mathrm{Pop}_{0}$ with a size of $N$. In GA, the term genotype refers to a candidate solution for a problem, which is often encoded as a bit string, while the phenotype is a domain solution itself and is encoded to be a genotype [26]. This process can be carried out randomly or can be guided by domain information.

2) Evaluation: This step involves calculating the fitness value of each individual in the population Pop $_{t}$.

3) Selection: This step involves applying a selection operator to the population Pop $_{t}$.

4) Elitism: This step involves selecting and preserving the elitist individual in the population.

5) Crossover: This step involves applying a crossover operator to the population $\mathrm{Pop}_{t}$.

6) Mutation: This step involves applying a mutation operator to the population $\mathrm{Pop}_{t}$ and creating the next generation's population $P_{o p_{t+1}}$.

7) Termination test: This step involves checking whether a satisfactory solution has been found, or the preset termination condition is met; if one of the condition is true, the algorithm is terminated. Otherwise, the procedure loops back to step (2).

This generic procedure of GA is illustrated as a flowchart in Figure 2.

A variety of implementations of GA have been researched and developed during the past few decades, and many of them are recognized as applicable and 


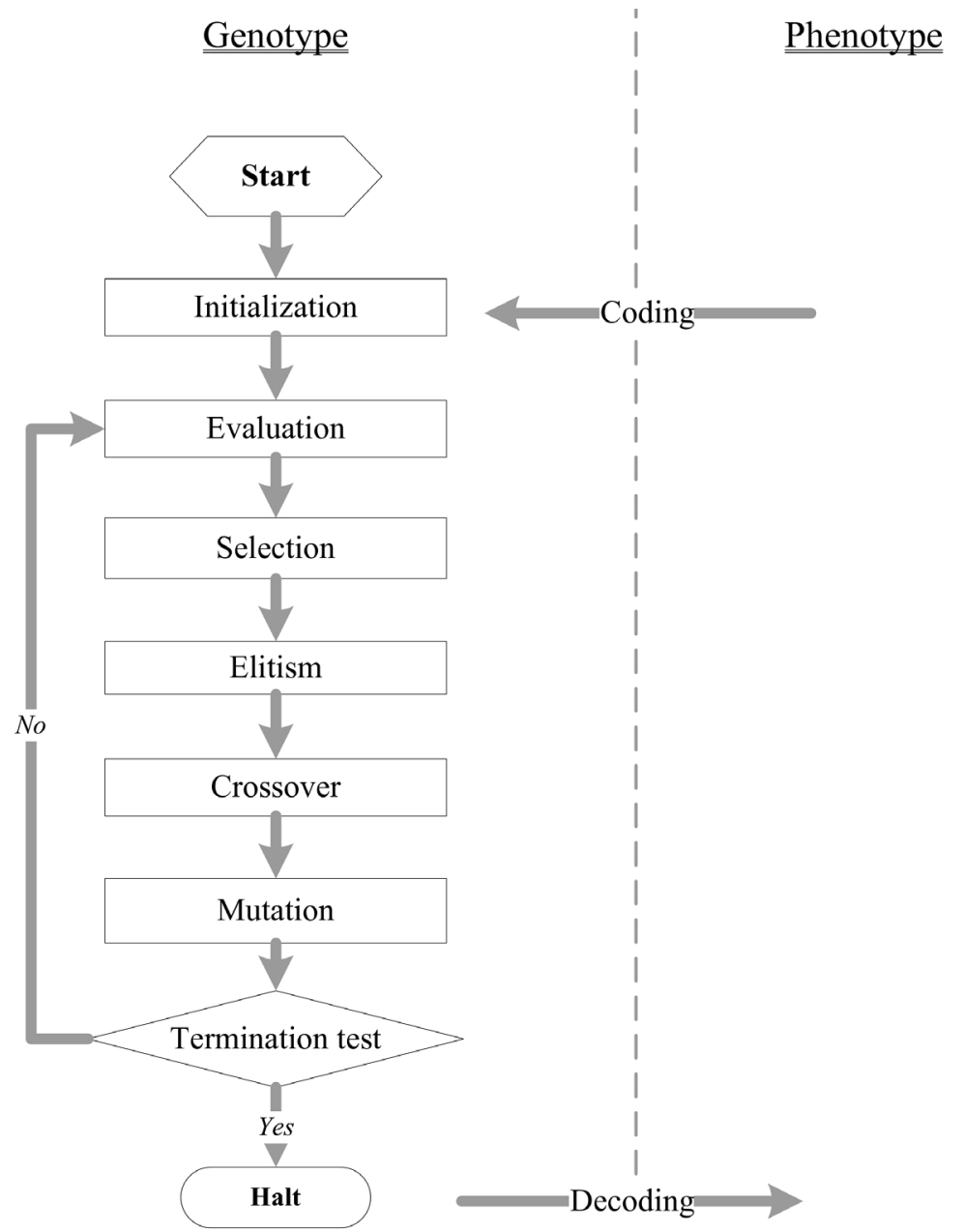

Figure 2. Flowchart of procedure of GA.

efficient for engineering applications. Among these implementations, the Genetic Algorithm Solver of the Global Optimization Toolbox (GASGOT) implements a simulated evolution in the Matlab ${ }^{\mathrm{TM}}$ (Trademark of MathWord) environment by using both binary and floating-point representations. GASGOT was developed by the Department of Industrial Engineering of North Carolina State University. This implementation provides a flexible platform of genetic operators, selection functions, termination functions, and the evaluation [27]. GASGOT runs in the Matlab ${ }^{\mathrm{TM}}$ workspace and can be easily invoked by other programs.

GASGOT supports both binary and floating-point representations, and the corresponding genetic operators have been developed. This study adopts GASGOT as the implementation tool of GA, and the applications and numeric examples were calculated in Matlab $^{\mathrm{TM}}$ based on the GA non-linear program (GANLP) solver engine of GASGOT.

\subsubsection{GA for Problem Solving of Non-Linear Problems (GANLP)}

A general non-linear programming problem (NLP) can be expressed as follows [28]:

$$
\operatorname{Max} z=f\left(x_{1}, x_{2}, \cdots, x_{n}\right)
$$




$$
\begin{gathered}
\text { s.t. } g_{1}\left(x_{1}, x_{2}, \cdots, x_{n}\right)(\leq \text {, or }=) b_{1} \\
g_{2}\left(x_{1}, x_{2}, \cdots, x_{n}\right)(\leq \text {, or }=) b_{2} \\
\cdots \\
g_{m}\left(x_{1}, x_{2}, \cdots, x_{n}\right)(\leq \text {, or }=) b_{m}
\end{gathered}
$$

[28] indicated that some specially formed non-linear programming problems can be solved by calculus-based algorithms, which assume that the objective function $f(x)$ and all non-linear constraints are twice continuously differentiable functions of $x$. Most calculus-based methods aim to transform the nonlinear problem into a sequence of solvable sub-problems. The methods generally require explicit or implicit second derivative calculations of the objective function, which in some of the methods can be ill-conditioned and can cause the algorithm to fail [29]. This weakness in the calculus-based method has prompted many researchers to propose GA, which is a random search method, for solving non-linear programming problems [30] [31].

The following example is taken from [28]:

$$
\begin{gathered}
\operatorname{Max} z=x_{1}\left(30-x_{1}\right)+x_{2}\left(35-x_{2}\right)-x_{1}^{2}-2 x_{2}^{2} \\
\text { s.t. } x_{1}^{2}+2 x_{2}^{2} \leq 250 \\
x_{1}+x_{2} \leq 20 \\
x_{1}, x_{2} \geq 0 .
\end{gathered}
$$

This problem, similar to many NLPs, can be formulated as follows:

$$
\begin{gathered}
\operatorname{Max} z=\sum_{j=1}^{n} f_{j}\left(x_{j}\right) \\
\text { s.t. } \sum_{j=i}^{n} g_{i j}\left(x_{j}\right) \leq b_{i}(i=1,2, \cdots, m) .
\end{gathered}
$$

Since the decision variables appear separate in terms of the objective function and the constraints, NLPs of this form are called separable programming problems. This kind of NLP can be solved by approximating each $f_{j}\left(x_{j}\right)$ and $g_{i j}\left(x_{j}\right)$ using a piecewise linear function [28].

The approximating problem for the above example Equation (4) gives the result $x_{1}=5, x_{2}=5, z=200$, while the actual optimal solution is $x_{1}=7.5, x_{2}=5.83, z=214.58$.

A GA program for the above example has been constructed with the parameters specified in Table 1.

The result given by the GA program is: $x_{1}=7.499, \quad x_{2}=5.8352, \quad z=214.58$. The evolution curve is shown as Figure 3. Compared to the results generated using the calculus-based method of [28], the GA generated results are closer to the actual optimal solution.

In Figure 3, it can be seen that at approximately the 15th generation, the optimal solution was generated. Although the fitness of the initial population shown in Figure 4 is not excellent, compared to the separable programming technique described in [28], the methods implemented in the GA non-linear problem solution engine of GASGOT are demonstrated to be straightforward, universal, efficient, and are feasible approaches for solving non-linear programming problems. 
Table 1. Parameters of genetic algorithms.

\begin{tabular}{cc}
\hline Representation scheme & Floating-point representation \\
\hline Selection operator & $\begin{array}{c}\text { Normalized geometric ranking } \\
\text { selection with rate 0.08, Elitism strategy } \\
\text { Heuristic crossover, arithmetic crossover } \\
\text { Crossover operator } \\
\text { Population size }\end{array}$ \\
Termination condition & 100 \\
\hline
\end{tabular}

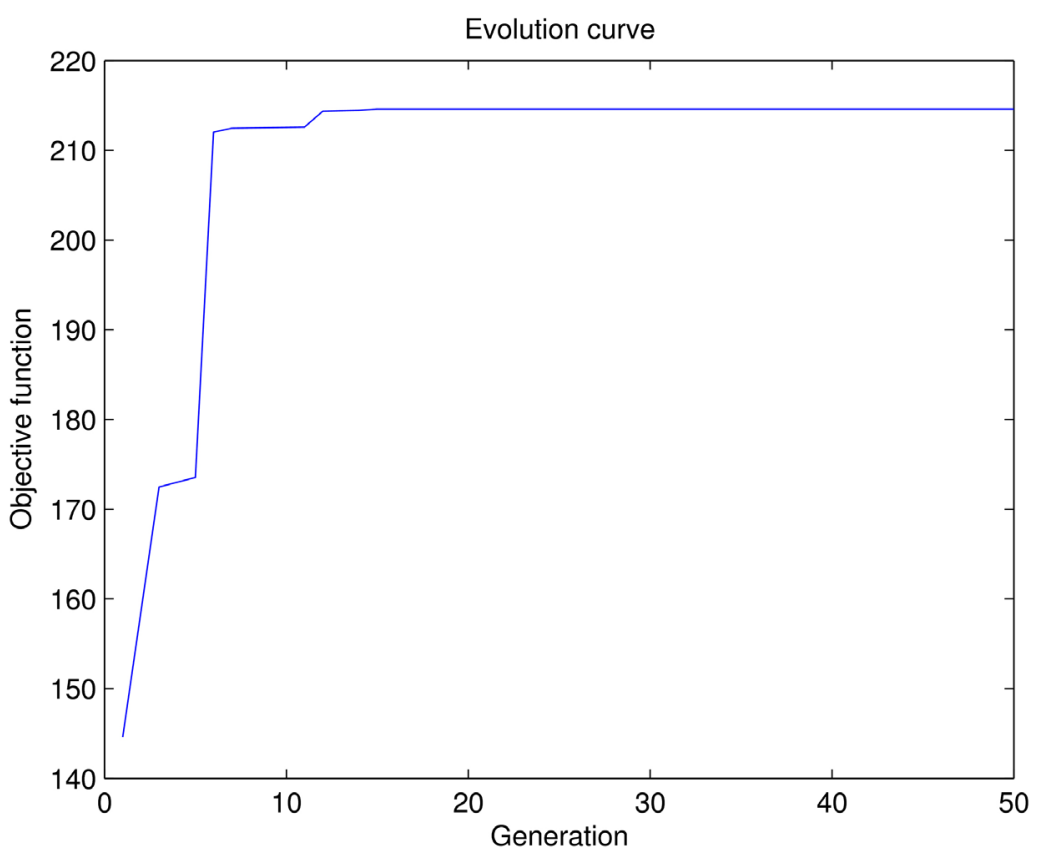

Figure 3. Evolution curve.

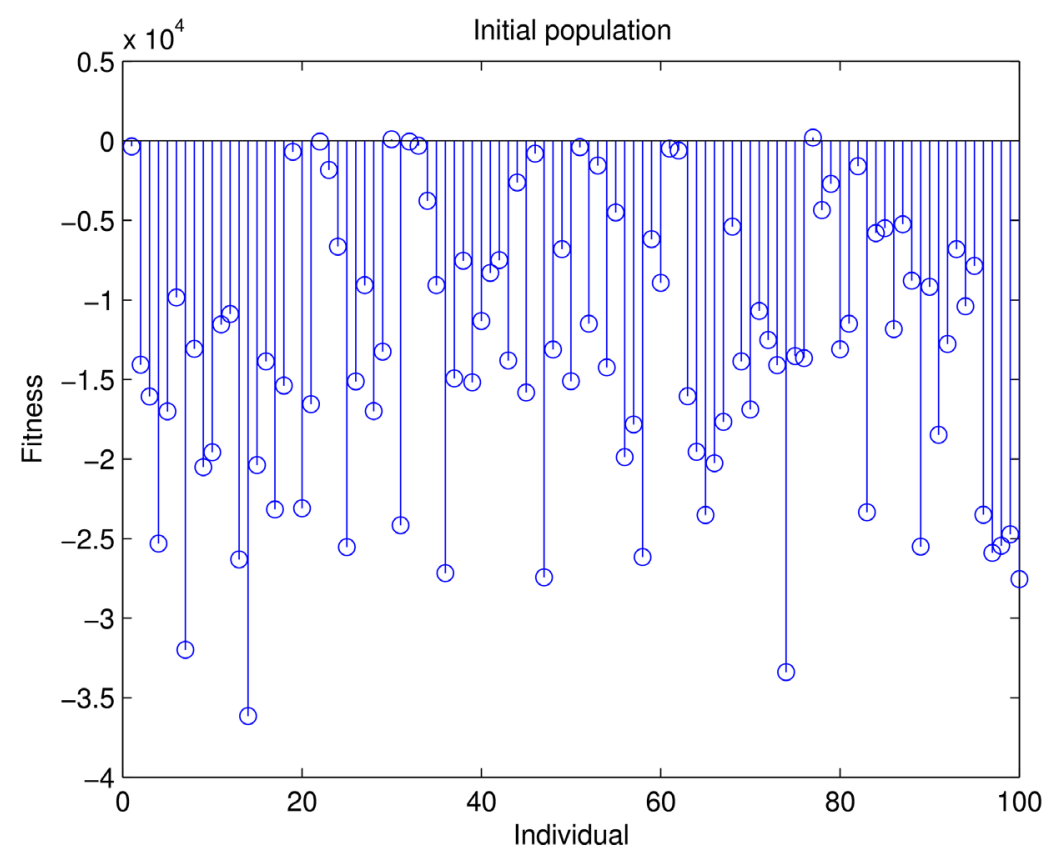

Figure 4. Fitness of initial population. 


\section{GA for Problem Solving of Inexact Non-Linear Problems (GAINLP)}

Quadratic programming problems are specific cases of non-linear programming problems [23]. Due to the lack of generally applicable algorithms for handling the non-linear structure, and the inexact information embedded in the structure, most non-linear programming problems are difficult to solve. The engine for solving interactive binary analysis based inexact linear problems proposed in [21] [22] is not intended for dealing with generic non-linear problems. By contrast, GA can be used as a general problem solver for this type of problems because there is not much difference for GA between treating the term of $x_{i}^{2}$ in quadratic programming problems and the terms $x_{i} x_{j}$ or $x_{i}^{0.28}$ in generic non-linear programming problems.

[23] has proposed a method for solving the IQP problem, which can be modified to solve generic inexact non-linear programming problems. The GA-based inexact non-linear programming method GAINLP involves three stages of problem solving. In the following, a computation experiment will be conducted to illustrate how the GAINLP method can handle complicated inexact non-linear problems Equation (6).

$$
\begin{gathered}
\operatorname{Max} f^{ \pm}=c_{1}^{ \pm} x_{1}^{ \pm}-c_{2}^{ \pm}\left(x_{1}^{ \pm}\right)^{0.3}-d_{1}^{ \pm} x_{2}^{ \pm}+d_{2}^{ \pm}\left(x_{1}^{ \pm} x_{2}^{ \pm}\right) \\
\text {s.t. } a_{11}^{ \pm}\left(x_{1}^{ \pm}\right)^{0.5}+a_{12}^{ \pm} x_{2}^{ \pm} \leq b_{1}^{ \pm} \\
x_{1}^{ \pm}+a_{2}^{ \pm} x_{2}^{ \pm} \leq b_{2}^{ \pm} \\
x_{j}^{ \pm} \geq 0, j=1,2
\end{gathered}
$$

where $a_{i j}^{ \pm}, b_{i}^{ \pm}, c_{j}^{ \pm}, d_{j}^{ \pm}$are inexact parameters and $x_{j}^{ \pm}$is an inexact variable, for an inexact number $g^{ \pm} \in\left[g^{-}, g^{+}\right], g^{+}$and $g^{-}$are the upper and lower bounds, respectively. In this experiment,

$$
\begin{gathered}
{\left[c_{1}^{-}, c_{1}^{+}\right]=[16,18] ;\left[c_{2}^{-}, c_{2}^{+}\right]=[12,14] ;\left[d_{1}^{-}, d_{1}^{+}\right]=[4,5] ;} \\
{\left[d_{2}^{-}, d_{2}^{+}\right]=[14,15] ;\left[a_{11}^{-}, a_{11}^{+}\right]=[4.5,5.5] ;\left[a_{12}^{-}, a_{12}^{+}\right]=[1.8,2.2] ;} \\
{\left[b_{1}^{-}, b_{1}^{+}\right]=[1.8,2.1] ;\left[a_{2}^{-}, a_{2}^{+}\right]=[1.8,2.2] ;\left[b_{2}^{-}, b_{2}^{+}\right]=[0.9,1.1] .}
\end{gathered}
$$

GAINLP has been designed to include three stages of problem solving.

In stage one, to obtain the initial suboptimal $x_{j}^{s}$, the random numbers of $a_{i j}^{r}, b_{i}^{r}, c_{j}^{r}, d_{j}^{r}$ were selected to transfer this INLP problem to a NLP problem, such that $a_{i j}^{r}, b_{i}^{r}, c_{j}^{r}, d_{j}^{r}$ satisfy the continuous uniform distribution in the intervals of $\left[a_{i j}^{-}, a_{i j}^{+}\right],\left[b_{i}^{-}, b_{i}^{+}\right],\left[c_{j}^{-}, c_{j}^{+}\right]$and $\left[d_{j}^{-}, d_{j}^{+}\right]$.

$$
\begin{gathered}
\operatorname{Max} f^{s}=c_{1}^{r} x_{1}^{s}-c_{2}^{r}\left(x_{1}^{s}\right)^{0.3}-d_{1}^{r} X_{2}^{s}+d_{2}^{r}\left(x_{1}^{s} x_{2}^{s}\right) \\
\text { s.t. } a_{11}^{r}\left(x_{1}^{s}\right)^{0.5}+a_{12}^{r} x_{2}^{s} \leq b_{1}^{r} \\
x_{1}^{s}+a_{2}^{r} x_{2}^{s} \leq b_{2}^{r} \\
x_{j}^{s} \geq 0, j=1,2 .
\end{gathered}
$$


Then, the heuristic search algorithm of the GANLP solver engine, presented in Section 2.2, can be used to identify a suboptimal solution $f^{s}$, and the corresponding decision variable $x_{j}^{s}$. The objective function in Equation (7) was used as the positive term of the fitness function and the constraints of Equation (6) adopted as the negative punishment terms. The result is:

$$
x_{1}^{s}=0.346, x_{2}^{s}=0.171, f^{s}=-2.296 .
$$

In stage two, by substituting $x_{1}^{s}, x_{2}^{s}$ into Equation (6), the inexact coefficients of $a_{i j}^{ \pm}, b_{i}^{ \pm}, c_{j}^{ \pm}, d_{j}^{ \pm}$will be determined. There are two kinds of decision schemes for inexact programming problems, the conservative scheme and optimistic scheme [20]. The former assumes less risk than the latter, so that for a maximization objective function, planning for the lower bound of an objective value $f^{-}$represents the conservative scheme, and planning for the upper bound of an objective value $f^{+}$represents the optimistic scheme [20]. In terms of constraints, the conservative scheme involves more rigorous or stringent constraints, and the optimistic scheme adopts more tolerant ones.

The $x_{1}^{s}, x_{2}^{s}$ obtained in stage one are used to construct two optimization problems in order to determine the coefficients of $a_{i j}^{ \pm+}, b_{i}^{ \pm+}, c_{j}^{ \pm+}, d_{j}^{ \pm+}$and $a_{i j}^{ \pm-}, b_{i}^{ \pm-}, c_{j}^{ \pm-}, d_{j}^{ \pm-}$respectively. The coefficients from the first group are considered to be corresponding to the optimistic scheme $f^{+}$, while the second group correspond to the conservative scheme $f^{-}$. Considering $c_{j}^{ \pm}, d_{j}^{ \pm}$are variables, the following two functions can be constructed:

$$
\begin{aligned}
\operatorname{Max} f^{+}=c_{1}^{ \pm+} x_{1}^{s}-c_{2}^{ \pm+} & \left(x_{1}^{s}\right)^{0.3}-d_{1}^{ \pm+} x_{2}^{s}+d_{2}^{ \pm+}\left(x_{1}^{s} x_{2}^{s}\right) \\
\text { s.t. } c_{1}^{ \pm+} & \in[16,18] \\
c_{2}^{ \pm+} & \in[12,14] \\
d_{1}^{ \pm+} & \in[4,5] \\
& d_{2}^{ \pm+} \in[14,15]
\end{aligned}
$$

and

$$
\begin{aligned}
\operatorname{Min} f^{-}=c_{1}^{ \pm-} X_{1}^{s}- & c_{2}^{ \pm-}\left(x_{1}^{s}\right)^{0.3}-d_{1}^{ \pm-} X_{2}^{s}+d_{2}^{ \pm-}\left(x_{1}^{s} x_{2}^{s}\right) \\
\text { s.t. } c_{1}^{ \pm-} & \in[16,18] \\
& c_{2}^{ \pm-} \in[12,14] \\
& d_{1}^{ \pm-} \in[4,5] \\
& d_{2}^{ \pm-} \in[14,15]
\end{aligned}
$$

To determine $a_{i j}^{ \pm^{+}}, b_{i}^{ \pm^{+}}$of the optimistic scheme in correspondence with the upper limit of the objective value $f^{+}$, the objective function can be constructed as follows:

$$
\begin{gathered}
\operatorname{Max} a b s\left(a_{11}^{ \pm}\left(x_{1}^{s}\right)^{0.5}+a_{12}^{ \pm} x_{2}^{s}-b_{1}^{ \pm}\right) \\
\text {s.t. } a_{11}^{ \pm}\left(x_{1}^{s}\right)^{0.5}+a_{12}^{ \pm} x_{2}^{s} \leq b_{1}^{ \pm}
\end{gathered}
$$

and 


$$
\begin{aligned}
& \operatorname{Max} a b s\left(x_{1}^{s}+a_{2}^{ \pm} x_{2}^{s}-b_{2}^{ \pm}\right) \\
& \text {s.t. } x_{1}^{s}+a_{2}^{ \pm} x_{2}^{s} \leq b_{2}^{ \pm},
\end{aligned}
$$

The objective functions to get $a_{i j}^{ \pm^{-}}, b_{i}^{ \pm^{-}}$of the conservative scheme are:

$$
\begin{gathered}
\text { Min } a b s\left(a_{11}^{ \pm}\left(x_{1}^{s}\right)^{0.5}+a_{12}^{ \pm} x_{2}^{s}-b_{1}^{ \pm}\right) \\
\text {s.t. } a_{11}^{ \pm}\left(x_{1}^{s}\right)^{0.5}+a_{12}^{ \pm} x_{2}^{s} \leq b_{1}^{ \pm}
\end{gathered}
$$

and

$$
\begin{aligned}
& \operatorname{Min} a b s\left(x_{1}^{s}+a_{2}^{ \pm} x_{2}^{s}-b_{2}^{ \pm}\right) \\
& \text {s.t. } x_{1}^{s}+a_{2}^{ \pm} x_{2}^{s} \leq b_{2}^{ \pm} .
\end{aligned}
$$

By solving the above functions Equations (8)-(11), the values of all the inexact coefficients are obtained, such that,

$$
\begin{aligned}
a_{11}^{ \pm^{+}}= & 4.5, a_{12}^{ \pm^{+}}=1.8, b_{1}^{ \pm^{-}}=2.1, a_{2}^{ \pm^{+}}=1.8, b_{2}^{ \pm^{+}}=1.1 \\
a_{11}^{ \pm^{-}}= & 5.5, a_{12}^{ \pm^{-}}=2.2, b_{1}^{ \pm^{-}}=1.8, a_{2}^{ \pm^{-}}=2.2, b_{2}^{ \pm^{-}}=0.9 \\
& c_{1}^{ \pm+}=18, c_{2}^{ \pm+}=12, d_{1}^{ \pm+}=4, d_{2}^{ \pm+}=15 ; \\
& c_{1}^{ \pm-}=16, c_{2}^{ \pm-}=14, d_{1}^{ \pm-}=5, d_{2}^{ \pm-}=14 .
\end{aligned}
$$

In stage three, the objective function presented in Equation (7) is converted into the following two sub-problems:

$$
\begin{gathered}
\operatorname{Max} f^{+}=18 x_{1}^{ \pm}-12\left(x_{1}^{ \pm}\right)^{0.3}-4 x_{2}^{ \pm}+15\left(x_{1}^{ \pm} x_{2}^{ \pm}\right), \\
\text {s.t. } 4.5\left(x_{1}^{ \pm}\right)^{0.5}+1.8 x_{2}^{ \pm} \leq 2.1, \\
x_{1}^{ \pm}+1.8 x_{2}^{ \pm} \leq 1.1, \\
x_{1}^{ \pm} \geq 0, x_{2}^{ \pm} \geq 0,
\end{gathered}
$$

and

$$
\begin{gathered}
\operatorname{Max} f^{-}=16 x_{1}^{ \pm}-14\left(x_{1}^{ \pm}\right)^{0.3}-5 x_{2}^{ \pm}+14\left(x_{1}^{ \pm} x_{2}^{ \pm}\right), \\
\text {s.t. } 5.5\left(x_{1}^{ \pm}\right)^{0.5}+2.2 x_{2}^{ \pm} \leq 1.8, \\
x_{1}^{ \pm}+2.2 x_{2}^{ \pm} \leq 0.9, \\
x_{1}^{ \pm} \geq 0, x_{2}^{ \pm} \geq 0 .
\end{gathered}
$$

In this stage, the inexact parameters in Equation (7) have been eliminated, and two typical non-linear optimization problems have been generated instead. Solving the above two objective functions by GANLP, the solution of the example Equation (6) is:

$$
f^{ \pm}=[-5.5575,-1.72], \quad x_{1}^{ \pm}=[0.24727,0.38496] \text {, and } x_{2}^{ \pm}=[0.1989,0.2053] \text {. }
$$

As demonstrated above, it can be seen that the GAINLP method can generate the optimal result without any simplification or assumption, and it can be adapted for applications of optimization problems with uncertainty. In the next section, this method will be applied on a real world regional waste management problem. 


\section{Case Study}

The study region includes three municipalities, a waste-to-energy (WTE) facility and a landfill, as shown in Figure 5. Three time periods are considered; each has an interval of five years. Over the 15 year planning horizon, an existing landfill and WTE facilities are available to serve the municipal solid waste (MSW) disposal needs in the region. The landfill has an existing capacity of $[2.05,2.30] \times 10^{6} \mathrm{t}$, and the WTE facility has a capacity of $[500,600] \mathrm{t} / \mathrm{d}$. The WTE facility generates residues of approximately $30 \%$ (on a mass basis) of the incoming waste streams, and its revenue from energy sale is $[15,25] \$ / \mathrm{t}$ combusted.

Table 2 shows the waste generation rates of the three municipalities and the operating costs of the two facilities in the three periods.

Taking into consideration the effects of the ES, the inexact non-linear (INLP) model can be formulated as follows:

$$
\begin{aligned}
& \operatorname{Min} f^{ \pm}= \sum_{i=1}^{2} \sum_{j=1}^{3} \sum_{k=1}^{3} L_{k} x_{i j k}^{ \pm}\left[A_{r e_{i j k}}^{ \pm}+C_{r e_{i j k}}^{ \pm}\left(x_{i j k}^{ \pm} / X_{r e_{i j k}}^{ \pm}\right)^{1+m}+\left(O P_{i k}^{ \pm}\right)\right] \\
&+\sum_{k=1}^{3} L_{k}\left(F E^{*} \sum_{j=1}^{3}\left(x_{2 j k}^{ \pm}\right)\right)\left\{A_{r e W T E-L F_{k}}\right. \\
&\left.+C_{r e W T E-L F_{k}}\left(F E^{*} \sum_{j=1}^{3} x_{2 j k}^{ \pm} / X_{r e W T E-L F_{k}}\right)^{1+m}+\left(O P_{1 k}^{ \pm}\right)\right\}-\sum_{k=1}^{3} \sum_{j=1}^{3} x_{2 j k}^{ \pm} R E_{k}^{ \pm} \\
& \text {S.t. } \sum_{j=1}^{3} \sum_{k=1}^{3} L_{k}\left[x_{1 j k}^{ \pm}+x_{2 j k}^{ \pm} F E\right] \leq T L^{ \pm} \sum_{j=1}^{3} x_{2 j k}^{ \pm} \leq T E^{ \pm}, \forall k \\
& \sum_{i=1}^{2} X_{i j k}=W G_{j k}^{ \pm}, \forall j, k \\
& X_{i j k}^{ \pm} \geq 0, \forall i, j, k
\end{aligned}
$$

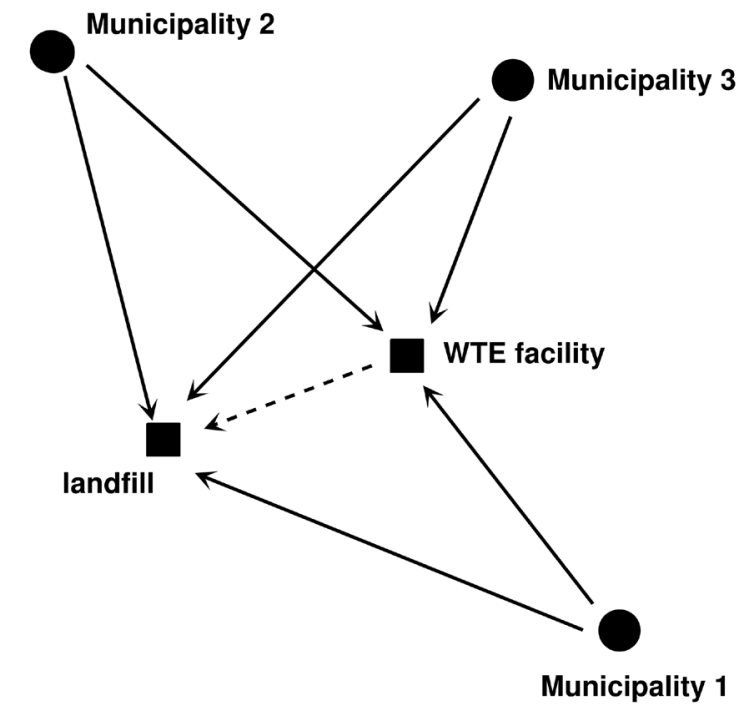

$\longrightarrow$ municipal solid waste

residue from WTE facility

Figure 5. Case study of municipalities and waste management facilities. 
Table 2. Data for the waste generation and treatment/disposal.

\begin{tabular}{cccc}
\hline \multicolumn{4}{c}{ Time Period } \\
\hline & Waste generation & $W G_{j k}^{ \pm}(\mathrm{t} / \mathrm{d})$ & $k=3$ \\
\hline Municipality $1(j=1)$ & {$[260,340]$} & {$[310,390]$} & {$[360,440]$} \\
Municipality $2(j=2)$ & {$[160,240]$} & {$[185,265]$} & {$[210,290]$} \\
Municipality $3(j=3)$ & {$[260,340]$} & {$[260,340]$} & {$[310,390]$} \\
\hline & Operation cost & OP $_{i k}^{ \pm}(\$ / \mathrm{t})$ & \\
\hline Landfill $(i=1)$ & {$[30,45]$} & {$[40,60]$} & {$[50,80]$} \\
WTE Facility $(i=2)$ & {$[55,75]$} & {$[60,85]$} & {$[65,95]$}
\end{tabular}

where, $i$ is the type of waste management facility ( $i=1,2$, where $i=1$ for landfill, 2 for WTE); $j$ is the city, $j=1,2,3 ; k$ is the time period, $k=1,2,3 ; L k$ is the length of period $k, L 1=L 2=L 3=365 * 5$ (day); $O P_{i k}^{ \pm}$is the operating cost of facility iduring period $k(\$ / \mathrm{t}) ; R E_{k}^{ \pm}$is the revenue from WTE during period $k \quad(\$ / \mathrm{t}), \quad R E_{1}^{ \pm}=R E_{2}^{ \pm}=R E_{3}^{ \pm}=[15,25] ; T E^{ \pm}$is the capacity of WTE $(\mathrm{t} / \mathrm{d}) ; T L^{ \pm}$is the capacity of the landfill $(\mathrm{t}) ; W G_{j k}^{ \pm}$is the waste disposal demand in city $j$ during period $k(\mathrm{t} / \mathrm{d}) ; x_{i j k}^{ \pm}$is the waste flow from city $j$ to facility $i$ during period $k(\mathrm{t} / \mathrm{d})$.

In this objective function Equation (12), the first term on the right side reflects the transportation costs in each management period ( $k=1$ to 3 ) from each city to each waste treatment unit, and the related operation costs. The second term reflects the cost incurred in transporting the products from the WTE facility to the landfill, and the operation cost at the landfill. The third term is the revenue generated from the WTE facility.

The MSW generation rates generally vary between different municipalities and for different periods, and the costs for the waste transportation and treatment also vary temporally and spatially. Furthermore, interactions exist between the waste flows and their transportation costs due to the effects of the ES. Tables 3-5 show the parameters related to the economy of scale, which include the fixed unit transportation cost Are, the reference waste flow $X_{r e}$ and the coefficient Crecorresponding to $X_{r e}$.

Hence, it can be observed that the traditional methods of binary analysis cannot solve this problem without additional assumptions or simplifications. The following discussion will explain how traditional methods solve this problem by simplifying the non-linear effects of the ES.

Scenario (i) Letting $m=-1$, the effects of the ES are totally ignored. This converts the inexact non-linear programming (INLP) problem to an inexact linear programming (ILP) problem, and the GAILP method presented in [23] can solve the problem.

Scenario (ii) Assuming $-0.2<m<-0.1$, it is indicated that the non-linear relationships in Equation (12) can be approximated by grey quadratic functions within a predetermined degree of error. Thus, the non-linear programming 
Table 3. Fixed unit transportation costs.

\begin{tabular}{|c|c|c|c|}
\hline \multicolumn{4}{|c|}{ City-to-landfill fixed unit transportation cost $A_{r_{l, j k}}^{ \pm}(\$ / t)$} \\
\hline$A_{r_{e_{1, k}}}^{ \pm}$ & {$[14.58,19.40]$} & {$[16.04,21.34]$} & {$[17.64,23.48]$} \\
\hline$A_{r_{e_{21} k}}^{ \pm}$ & {$[12.65,16.87]$} & {$[13.92,18.56]$} & {$[15.31,20.41]$} \\
\hline$A_{r_{13 k}}^{ \pm}$ & {$[15.30,20.49]$} & {$[16.83,22.53]$} & {$[18.52,24.79]$} \\
\hline \multicolumn{4}{|c|}{ City-to-WTE fixed unit transportation cost $A_{r_{2, j}}^{ \pm}(\$ / t)$} \\
\hline$A_{r_{21 k}}^{ \pm}$ & {$[11.57,15.42]$} & {$[12.73,16.97]$} & {$[14.00,18.66]$} \\
\hline$A_{r e_{22 k}}^{ \pm}$ & {$[12.17,16.15]$} & {$[13.39,17.76]$} & {$[14.73,19.54]$} \\
\hline$A_{r e_{33 k}}^{ \pm}$ & {$[10.60,14.10]$} & {$[11.67,15.51]$} & {$[12.83,17.06]$} \\
\hline \multicolumn{4}{|c|}{ WTE-to-landfill fixed unit transportation cost $A_{\text {reWTE-LF }}^{ \pm}(\$ / \mathrm{t})$} \\
\hline & {$[5.71,7.62]$} & {$[6.28,8.38]$} & {$[6.91,9.33]$} \\
\hline
\end{tabular}

Table 4. Reference waste flow.

\begin{tabular}{|c|c|c|c|}
\hline \multicolumn{4}{|c|}{ City-to-landfill reference waste flow $X_{r_{e_{j, k}}}^{ \pm}(\mathrm{t} / \mathrm{d})$} \\
\hline$X_{r_{e_{11}}}^{ \pm}$ & {$[220,250]$} & {$[240,280]$} & {$[260,320]$} \\
\hline$X_{r_{12 k}}^{ \pm}$ & {$[160,200]$} & {$[180,220]$} & {$[220,260]$} \\
\hline$X_{r_{13 k}}^{ \pm}$ & {$[160,200]$} & {$[180,240]$} & {$[200,240]$} \\
\hline \multicolumn{4}{|c|}{ City-to-WTEreference waste flow $X_{r_{2, k}}^{ \pm}(\mathrm{t} / \mathrm{d})$} \\
\hline$X_{r_{e_{11}}}^{ \pm}$ & {$[200,240]$} & {$[240,280]$} & {$[280,320]$} \\
\hline$X_{r_{22 k}}^{ \pm}$ & {$[120,170]$} & {$[150,190]$} & {$[180,220]$} \\
\hline$X_{r_{23 k}}^{ \pm}$ & {$[220,270]$} & {$[220,270]$} & {$[240,270]$} \\
\hline \multicolumn{4}{|c|}{ WTE-to-landfill reference waste flow $X_{\text {reWTE-LLEk }}^{ \pm}(\mathrm{t} / \mathrm{d})$} \\
\hline & {$[170,200]$} & {$[200,260]$} & {$[240,270]$} \\
\hline
\end{tabular}

Table 5. $C_{r e}(\$ / \mathrm{t})$ The coefficient representing the economy of scale corresponding to reference waste flow $X_{r e}$.

\begin{tabular}{|c|c|c|c|c|c|c|c|}
\hline & $k=1$ & $k=2$ & $k=3$ & & $k=1$ & $k=2$ & $k=3$ \\
\hline$C_{r e_{1 k}}^{-}$ & -2.7 & -3.4 & -3.8 & $C_{r e_{21 k}}^{-}$ & -1.9 & -2.6 & -3.3 \\
\hline$C_{r e_{1 k}}^{+}$ & -4.1 & -5.0 & -6.3 & $C_{r_{21 k}}^{+}$ & -3.1 & -4.0 & -5.0 \\
\hline$C_{r_{12 k}}^{-}$ & -1.7 & -2.1 & -2.8 & $C_{r_{22 k}}^{-}$ & -1.2 & -1.7 & -2.2 \\
\hline$C_{r e_{12 k}}^{+}$ & -2.8 & -3.4 & -4.5 & $C_{r e_{22 k}}^{+}$ & -2.3 & -2.8 & -3.6 \\
\hline$C_{r_{13 k}}^{-}$ & -2.1 & -2.5 & -3.1 & $C_{r_{23 k}}^{-}$ & -2.0 & -2.2 & -2.6 \\
\hline$C_{r_{13 k}}^{+}$ & -3.4 & -4.5 & -5.0 & $C_{r_{23 k}}^{+}$ & -3.2 & -3.5 & -3.9 \\
\hline$C_{\text {reWTE-LLF }}^{-}$ & -0.8 & -1.1 & -1.4 & & & & \\
\hline$C_{\text {rWWTE-LIF }}^{+}$ & -1.3 & -1.8 & -2.1 & & & & \\
\hline
\end{tabular}

Note: The + and -superscript sign of $C_{r e}$ represents the value of $C_{r e}$ relevant to the upper and lower bound of $X_{\text {re }}$ only.

problem is converted into an inexact quadratic programming problem.

Table 6 lists the solutions of the objective function Equation (12) for above two scenarios (i) and (ii).

Both of the above simplifications introduced inaccuracy and limitations. When 
the value of $m$ deviates away from the predetermined value, this inaccuracy will increase dramatically.

Applying the GAINLP model on the inexact non-linear programming problem, the optimization problem represented in Equation (12) can be solved directly without additional assumptions for the effects of the ES. By adopting this approach, the solution can be found even when the ES exponent $\mathrm{m}$ is not within the interval of $[-0.2,-0.1]$.

Three different scenarios, $m=-0.1, m=-0.3, m=-0.5$ have been tested, and the solutions given by the GAINLP model are shown in Table 7.

The above scenarios assume that the economy of scale exponent is universal in the whole region during the entire period. However, this is not always necessarily true for practical engineering problems. More common situations may involve different scale exponents for various combinations of municipalities and facilities in different periods. Thus, Table 8 illustrates the solutions for the fourth scenario, which involves different scale exponents.

In the objective function of the inexact non-linear programming model Equation (12), the weight of the transportation cost in the system operation cost varies according to different $C_{t}$ values. The effect becomes significant when waste

Table 6. Solutions obtained by applying the ILP model $(m=-1)$ and IQP model $(-0.2<m<-0.1)$.

\begin{tabular}{|c|c|c|c|c|c|}
\hline $\begin{array}{l}\text { Decision } \\
\text { Variable }\end{array}$ & Facility & Municipality & Period & $\begin{array}{l}\text { ILP Solution }(\mathrm{t} / \mathrm{d}) \\
\qquad m=-1\end{array}$ & $\begin{array}{l}\text { IQP Solution (t/d) } \\
-0.2<m<-0.1\end{array}$ \\
\hline$x_{111}^{ \pm}$ & 1 & 1 & 1 & {$[210,290]$} & {$[250,290]$} \\
\hline$x_{112}^{ \pm}$ & 1 & 1 & 2 & 0 & {$[310,350]$} \\
\hline$x_{113}^{ \pm}$ & 1 & 1 & 3 & {$[0,30]$} & {$[360,440]$} \\
\hline$x_{121}^{ \pm}$ & 1 & 2 & 1 & 0 & {$[0,30]$} \\
\hline$X_{122}^{ \pm}$ & 1 & 2 & 2 & {$[0,65]$} & {$[185,225]$} \\
\hline$x_{123}^{ \pm}$ & 1 & 2 & 3 & {$[210,290]$} & {$[50,80]$} \\
\hline$x_{131}^{ \pm}$ & 1 & 3 & 1 & {$[0,30]$} & 0 \\
\hline$x_{132}^{ \pm}$ & 1 & 3 & 2 & {$[260,330]$} & 0 \\
\hline$x_{133}^{ \pm}$ & 1 & 3 & 3 & {$[170,200]$} & 0 \\
\hline$x_{211}^{ \pm}$ & 2 & 1 & 1 & 50 & {$[10,50]$} \\
\hline$x_{212}^{ \pm}$ & 2 & 1 & 2 & {$[310,390]$} & {$[0,40]$} \\
\hline$x_{213}^{ \pm}$ & 2 & 1 & 3 & {$[360,410]$} & 0 \\
\hline$x_{221}^{ \pm}$ & 2 & 2 & 1 & {$[160,240]$} & {$[160,210]$} \\
\hline$x_{222}^{ \pm}$ & 2 & 2 & 2 & {$[185,200]$} & {$[0,40]$} \\
\hline$x_{223}^{ \pm}$ & 2 & 2 & 3 & 0 & {$[160,210]$} \\
\hline$x_{231}^{ \pm}$ & 2 & 3 & 1 & {$[260,310]$} & {$[260,340]$} \\
\hline$x_{232}^{ \pm}$ & 2 & 3 & 2 & {$[0,10]$} & {$[260,340]$} \\
\hline$x_{233}^{ \pm}$ & 2 & 3 & 3 & {$[140,190]$} & {$[310,390]$} \\
\hline \multicolumn{4}{|c|}{ System cost $\left(\$ 10^{6}\right) \quad f^{ \pm}$} & {$[220.2,507.4]$} & {$[239.5,514.1]$} \\
\hline
\end{tabular}

Note: Facility: 1 = landfill, 2 = WTC Facility. 
Table 7. Solutions when $m=-0.1,0.3$ and 0.5 .

\begin{tabular}{|c|c|c|c|c|c|c|}
\hline $\begin{array}{l}\text { Decision } \\
\text { Variable }\end{array}$ & Facility & Munici-pality & Period & & Solution & \\
\hline & & (t/d) & & $m=-0.1$ & $m=-0.3$ & $m=-0.5$ \\
\hline$x_{111}^{ \pm}$ & 1 & 1 & 1 & {$[203,292]$} & {$[100,221]$} & {$[35,88]$} \\
\hline$x_{112}^{ \pm}$ & 1 & 1 & 2 & {$[1,36]$} & {$[1,44]$} & {$[1,36]$} \\
\hline$x_{113}^{ \pm}$ & 1 & 1 & 3 & {$[1,44]$} & {$[126,190]$} & {$[240,300]$} \\
\hline$x_{121}^{ \pm}$ & 1 & 2 & 1 & {$[1,43]$} & {$[60,141]$} & {$[144,240]$} \\
\hline$x_{122}^{ \pm}$ & 1 & 2 & 2 & {$[1,73]$} & {$[20,103]$} & {$[75,148]$} \\
\hline$x_{123}^{ \pm}$ & 1 & 2 & 3 & {$[200,290]$} & {$[200,259]$} & {$[197,260]$} \\
\hline$x_{131}^{ \pm}$ & 1 & 3 & 1 & {$[1,37]$} & {$[90,190]$} & {$[225,312]$} \\
\hline$x_{132}^{ \pm}$ & 1 & 3 & 2 & {$[247,332]$} & {$[189,270]$} & {$[120,200]$} \\
\hline$x_{133}^{ \pm}$ & 1 & 3 & 3 & {$[154,209]$} & {$[139,210]$} & {$[143,192]$} \\
\hline$x_{211}^{ \pm}$ & 2 & 1 & 1 & {$[35,58]$} & {$[120,167]$} & {$[220,307]$} \\
\hline$x_{212}^{ \pm}$ & 2 & 1 & 2 & {$[295,390]$} & {$[299,385]$} & {$[295,390]$} \\
\hline$x_{213}^{ \pm}$ & 2 & 1 & 3 & {$[329,426]$} & {$[202,323]$} & {$[120,161]$} \\
\hline$X_{221}^{ \pm}$ & 2 & 2 & 1 & {$[147,240]$} & {$[55,145]$} & {$[1,30]$} \\
\hline$x_{222}^{ \pm}$ & 2 & 2 & 2 & {$[165,222]$} & {$[142,200]$} & {$[80,154]$} \\
\hline$x_{223}^{ \pm}$ & 2 & 2 & 3 & {$[1,25]$} & {$[1,40]$} & {$[1,43]$} \\
\hline$x_{231}^{ \pm}$ & 2 & 3 & 1 & {$[230,320]$} & {$[122,164]$} & {$[12,40]$} \\
\hline$x_{232}^{ \pm}$ & 2 & 3 & 2 & {$[1,28]$} & {$[30,100]$} & {$[108,167]$} \\
\hline$x_{233}^{ \pm}$ & 2 & 3 & 3 & {$[125,200]$} & {$[125,194]$} & {$[120,214]$} \\
\hline \multicolumn{3}{|c|}{ System cost $\left(\$ 10^{6}\right)$} & $f^{ \pm}=$ & {$[209.8,522.3]$} & {$[200.5,519.6]$} & {$[197.6,516.8]$} \\
\hline
\end{tabular}

Note: Facility: 1 = landfill, 2 = WTC Facility.

flow becomes lower and the hauling distances are substantial. This effect is a nonlinear function of the waste flow $x_{i j k}$, in which the reference waste flow $x_{r e}$ and the economy of scale exponent $m$ are the parameters. Thus, the problem is a complicated non-linear programming problem, and the GA-based search approach has been shown to be adequate for solving this kind of economy optimization problems.

It is also reasonable to assume that between different locations ( $i=1,2 ; j=1,2,3)$, for different time periods $(k=1,2,3)$, the economy of scale exponent $\left(m_{i j k}\right.$ and $\left.m W T E-L F_{k}\right)$ may be different, since $m$ is the parameter used to describe the characteristics and attributes of a particular transportation scenario. Under these considerations, scenario 4 was designed with different operation costs and transportation strategies. On the other hand, the traditional inexact linear and inexact quadratic programming methods will not be able to handle situations like scenario 4 without additional assumptions and simplification.

The results also show that when the value of the economy of scale exponent $m$ becomes smaller, from $-0.1,-0.3$ to -0.5 , for both $f$ positive scheme and $f$ negative scheme, the value of the minimized objective function also becomes 
Table 8. Solutions when $m$ is different for each municipality and each period.

\begin{tabular}{|c|c|c|c|c|c|}
\hline Symbol & Facility & Municipality & Period & Solution & $m$ \\
\hline \multicolumn{6}{|c|}{ Decision Variable $(\mathrm{t} / \mathrm{d})$} \\
\hline$x_{111}^{ \pm}$ & landfill & 1 & 1 & {$[100,192]$} & -0.15 \\
\hline$x_{112}^{ \pm}$ & landfill & 1 & 2 & {$[0,40]$} & -0.2 \\
\hline$x_{113}^{ \pm}$ & landfill & 1 & 3 & {$[112,178]$} & -0.35 \\
\hline$x_{121}^{ \pm}$ & landfill & 2 & 1 & {$[65,139]$} & -0.2 \\
\hline $\mathrm{X}_{122}^{ \pm}$ & landfill & 2 & 2 & {$[40,78]$} & -0.3 \\
\hline$x_{123}^{ \pm}$ & landfill & 2 & 3 & {$[199,279]$} & -0.3 \\
\hline$x_{131}^{ \pm}$ & landfill & 3 & 1 & {$[90,189]$} & -0.25 \\
\hline$x_{132}^{ \pm}$ & landfill & 3 & 2 & {$[135,280]$} & -0.25 \\
\hline$x_{133}^{ \pm}$ & landfill & 3 & 3 & {$[127,218]$} & -0.3 \\
\hline$x_{211}^{ \pm}$ & WTC Facility & 1 & 1 & {$[125,189]$} & -0.1 \\
\hline$x_{212}^{ \pm}$ & WTC Facility & 1 & 2 & {$[300,390]$} & -0.15 \\
\hline$x_{213}^{ \pm}$ & WTC Facility & 1 & 3 & {$[205,312]$} & -0.15 \\
\hline$x_{221}^{ \pm}$ & WTC Facility & 2 & 1 & {$[42,142]$} & -0.45 \\
\hline$x_{222}^{ \pm}$ & WTC Facility & 2 & 2 & {$[129,192]$} & -0.3 \\
\hline$x_{223}^{ \pm}$ & WTC Facility & 2 & 3 & {$[0,40]$} & -0.1 \\
\hline$x_{231}^{ \pm}$ & WTC Facility & 3 & 1 & {$[100,198]$} & -0.3 \\
\hline$x_{232}^{ \pm}$ & WTC Facility & 3 & 2 & {$[44,113]$} & -0.45 \\
\hline \multirow[t]{2}{*}{$x_{233}^{ \pm}$} & WTC Facility & 3 & 3 & {$[99,219]$} & -0.4 \\
\hline & System cost $\left(\$ 10^{6}\right)$ & & $f^{ \pm}=$ & {$[194.7,500.1]$} & \\
\hline
\end{tabular}

Note: for transportation from WTE facility to landfill, $m=-0.5$.

smaller. At the same time, the range of the intervals of the minimized objective function also decreases. This reflects how the economy of scale exponent affects the overall cost for the entire period. A comparison of the results for the four scenarios is given in Figure 6.

\section{Conclusion}

This paper has proposed an innovative GA-based inexact non-linear programming problem solving approach (GAINLP). The GAINLP was applied to a solid waste management optimization problem, under different scenarios, and the results from the GAINLP, GAILP, and GAIQP methods were compared. The comparison illustrates the practicality and flexibility of the proposed GAINLP method for solving more complex inexact non-linear problems. Similar to other allpurpose heuristic search methods, this problem solving approach is widely adaptable for different problem scenarios that involve non-linear optimization and inexact information. The GAINLP method was implemented in Matlab, and it can be integrated with other operation programming software packages. The implementation of GAINLP can also be easily extended to include other non-linear programming solvers so as to enhance the flexibility and efficiency of the problem solving process. 


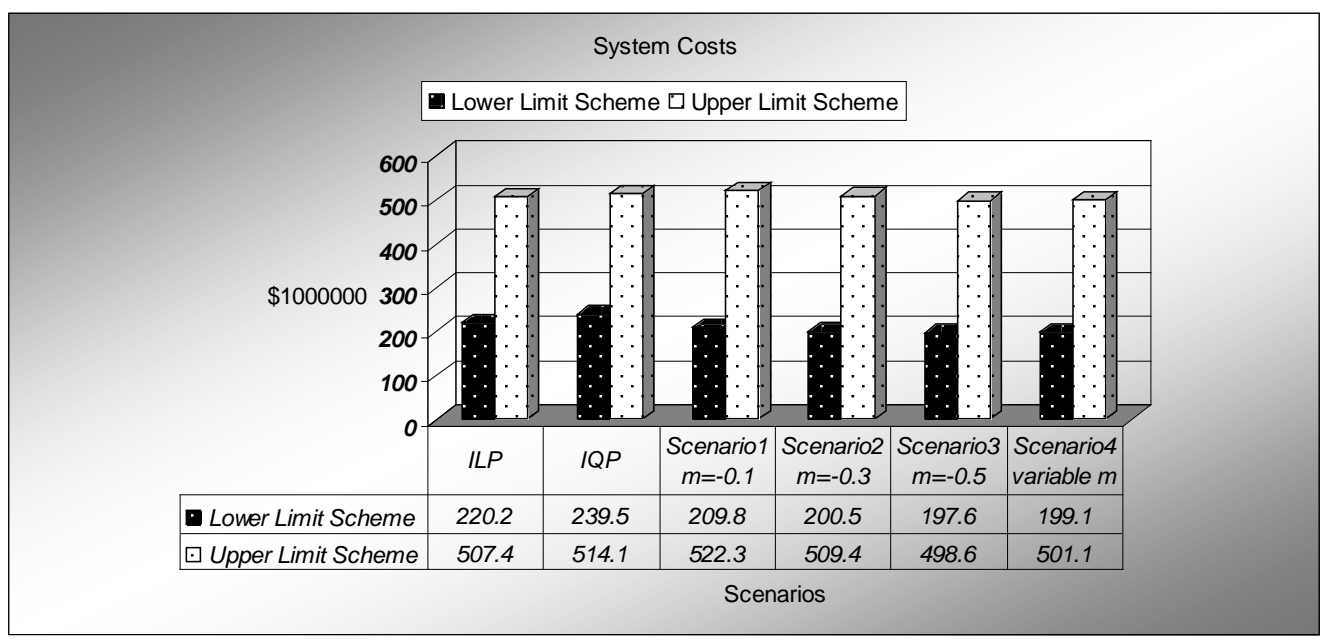

Figure 6. System cost comparisons.

\section{Acknowledgements}

The generous support of the Natural Sciences and Engineering Research Council (NSERC) and the Canada Research Chair Program of Canada are gratefully acknowledged.

\section{References}

[1] Anderson, L. (1968) A Mathematical Model for the Optimization of a Waste Management System. Technical Report 68-1, Sanitary Engineering Research Laboratory, University of California, Berkely.

[2] Christensen, H. and Haddix, G. (1974) A Model for Sanitary Landfill Management and Decision. Computers and Operation Research, 1, 275-281.

https://doi.org/10.1016/0305-0548(74)90052-5

[3] Fuertes, L., Hundson, J. and Mark, D. (1974) Solid Waste Management: Equity Trade-Off Models. Journal of Urban Planning and Development, 100, 155-171.

[4] Jenkins, L. (1982) Parametric Mixed Integer Programming: An Application to Solid Waste Management. Management Science, 28, 1271-1284.

https://doi.org/10.1287/mnsc.28.11.1270

[5] Jacobs, T. and Everett, J. (1992) Optimal Scheduling of Landfill Operations Incorporating Recycling. Journal of Environmental Engineering, 118, 420-429. https://doi.org/10.1061/(ASCE)0733-9372(1992)118:3(420)

[6] Badran, M. and El-Haggar, S. (2006) Optimization of Municipal Solid Waste Management in Port Said-Egypt. Waste Management, 26, 534-545.

https://doi.org/10.1016/j.wasman.2005.05.005

[7] Sushi, A. and Vart, P. (1989) Waste Management Policy Analysis and Growth Monitoring: An Integrated Approach to Perspective Planning. International Journal of Systems Science, 20, 907-926. https://doi.org/10.1080/00207728908910180

[8] Chang, N. (1996) A Grey Fuzzy Multiobjective Programming Approach for the Optimal Planning of a Reservoir Watershed, Part A: Theoretical Development. Water Research, 30, 2329-2340. https://doi.org/10.1016/0043-1354(96)00124-8

[9] Chang, N., Shoemaker, C. and Schuler, R. (1996) Solid Waste Management System Analysis with Air Pollution and Leachate Impact Limitations. Waste Management and Research, 14, 463-481. https://doi.org/10.1177/0734242X9601400505 
[10] Huang, G., Baetz, B. and Patry, G. (1995) Grey Integer Programming: An Application to Waste Management Planning under Uncertainty. European Journal of $O p$ erational Research, 83, 594-620. https://doi.org/10.1016/0377-2217(94)00093-R

[11] Huang, G., Baetz, B. and Patry, G. (1995) Grey Quadratic Programming and Its Application to Municipal Waste Management Planning under Uncertainty. Engineering Optimization, 23, 201-223. https://doi.org/10.1080/03052159508941354

[12] Li, Y. and Huang, G. (2009) Dynamic Analysis for Solid Waste Management Systems: An Inexact Multistage Integer Programming Approach. Journal of the Air and Waste Management Association, 59, 279-292. https://doi.org/10.3155/1047-3289.59.3.279

[13] Huang, G. and Cai, Y. (2010) A Superiority-Inferiority-Based Inexact Fuzzy Stochastic Programming Approach for Solid Waste Management under Uncertainty. Environmental Modeling and Assessment, 15, 381-396.

https://doi.org/10.1007/s10666-009-9214-6

[14] Ekmekçioglu, M., Kaya, T. and Kahraman, C. (2010) Fuzzy Multicriteria Disposal Method and Site Selection for Municipal Solid Waste. Waste Management, 30, 1729-1737. https://doi.org/10.1016/j.wasman.2010.02.031

[15] Piresa, A., Martinho, G. and Chang, N. (2001) Solid Waste Management in European Countries: A Review of Systems Analysis Techniques. Journal of Environmental Management, 92, 1033-1050. https://doi.org/10.1016/j.jenvman.2010.11.024

[16] Beliën, J., De Boeck, L. and Van Ackere, J. (2012) Municipal Solid Waste Collection and Management Problems: A Literature Review. Transportation Science, 48, 78102. https://doi.org/10.1287/trsc.1120.0448

[17] Or, I. and Curi, K. (1993) Improving the Efficiency of the Solid Waste Collection System in Izmir, Turkey, through Mathematical Programming. Waste Management \& Research, 11, 297-311. https://doi.org/10.1177/0734242X9301100404

[18] Sun, W., Huang, G., Lv, Y. and Li, G. (2013) Inexact Joint-Probabilistic ChanceConstrained Programming with Left-Hand-Side Randomness: An Application to Solid Waste Management. European Journal of Operational Research, 228, 217-225. https://doi.org/10.1016/j.ejor.2013.01.011

[19] Chang, N., Schuler, R. and Shoemaker, C. (1993) Environment and Economic Optimization of an Integrated Solid Waste Management System. Journal of Resource Management and Technology, 21, 87-100.

[20] Huang, G., Baetz, B. and Patry, G. (1993) A Grey Fuzzy Linear Programming Approach for Municipal Solid Waste Management Planning under Uncertainty. Civil Engineering Systems, 10, 123-146. https://doi.org/10.1080/02630259308970119

[21] Huang, G., Baetz, B. and Patry, G. (1994) Grey Dynamic Programming for Solid Waste Management Planning Under Uncertainty. Journal of Urban Planning and Development, 120, 132-156. https://doi.org/10.1061/(ASCE)0733-9488(1994)120:3(132)

[22] Huang, G., Baetz, B. and Patry, G. (1994) Waste Flow Allocation Planning through a Grey Fuzzy Quadratic Programming Approach. Civil Engineering Systems, 11, 209-243. https://doi.org/10.1080/02630259408970147

[23] Jin, W., Hu, Z. and Chan, C. (2013) A Genetic-Algorithms-Based Approach for Programming Linear and Quadratic Optimization Problems with Uncertainty. Mathematical Problems in Engineering, 2013, Article ID: 272491.

http://www.hindawi.com/journals/mpe/2013/272491/ https://doi.org/10.1155/2013/272491

[24] Chen, M. and Huang, G. (2001) A Derivative Algorithm for Inexact Quadratic Program-Application to Environmental Decision-Making under Uncertainty. Euro- 
pean Journal of Operational Research, 128, 570-586.

https://doi.org/10.1016/S0377-2217(99)00374-4

[25] Callan, S. and Thomas. J. (2001) Economies of Scale and Scope: A Cost Analysis of Municipal Solid Waste Services. Land Economics, 77, 548-560.

https://doi.org/10.2307/3146940

[26] Melanie. M. (1998) An Introduction of Genetic Algorithms, The MIT Press, Cambridge.

[27] Houck, C., Joines, J. and Kay, M. (1995) A Genetic Algorithm for Function Optimization: A Matlab Implementation. NCSU-IE TR 95.09.

[28] Winston, W. (2003) Operation Research: Applications and Algorithms. Duxbury Press, Boston.

[29] Michalewicz, Z. (1998) Genetic Algorithm + Data Structures = Evolution Programs. 3rd Revised and Extended Edition, Springer, Berlin.

[30] Gen, M. and Cheng, R. (1997) Genetic Algorithm and Engineering Design. Wiley, New York.

[31] Joines, J. and Houck, C. (1994) On the Use of Non-Stationary Penalty Functions to Solve Constrained Optimization Problems with Genetic Algorithms. Proceedings of the 1 st IEEE Conference on Evolutionary Computation, Orlando, 27-29 June 1994, 579-584.

Submit or recommend next manuscript to SCIRP and we will provide best service for you:

Accepting pre-submission inquiries through Email, Facebook, LinkedIn, Twitter, etc. A wide selection of journals (inclusive of 9 subjects, more than 200 journals)

Providing 24-hour high-quality service

User-friendly online submission system

Fair and swift peer-review system

Efficient typesetting and proofreading procedure

Display of the result of downloads and visits, as well as the number of cited articles

Maximum dissemination of your research work

Submit your manuscript at: http://papersubmission.scirp.org/

Or contact jep@scirp.org 